\title{
Evidence for a pion condensate formation in pp interactions at U-70
}

\author{
Elena Kokoulina ${ }^{* \dagger}$ \\ Joint Institute for Nuclear Research \\ E-mail: kokoulindsunse.jinr.ru
}

High multiplicity study (project Thermalization, experiment E-190) is carried out at U-70 accelerator at IHEP (Protvino, Russia). This project is aimed at the search for collective phenomena. It is known that mainly pions are produced at the $50 \mathrm{GeV}$-proton beam accelerator. Their mean energy is decreased with multiplicity increase. In that system Bose-Einstein condensate (BEC) can be formed. The theoretical and experimental studies of BEC are continued from 70es. M. Gorenstein and V. Begun have shown within the framework of an ideal pion gas model that sharp growth of the neutral pion number fluctuations will be a signal of BEC formation with the increase of total multiplicity (neutral and charged particle sum). SVD-2 Collaboration (JINR, IHEP and SINP MSU) investigated neutral pion number fluctuations in pp interactions at $50 \mathrm{GeV} / \mathrm{c}$ incident beam at U-70 versus total multiplicity and has revealed noticeable growth of scaled variance with the total multiplicity increase. The growth of these fluctuations reaches more than 7 standard deviations for the scaled variance at total multiplicity about 30 pions as opposed to the tendency for the simulated events. This growth has been observed both registered photons and restored neutral pions.

36th International Conference on High Energy Physics

4-11 July 2012

Melbourne, Australia

\footnotetext{
* Speaker.

${ }^{\dagger}$ On behalf of the SVD-2 Collaboration.
} 


\section{Introduction}

Experiments at LHC give evidence of a similarity of multiple production mechanisms in proton interactions at high multiplicities [U] and central collisions of relativistic heavy ions (RHIC). Studies at high multiplicity (more than mean value) region are carried out at U-70 accelerator at IHEP (Protvino). The experimental setup Spectrometer with Vertex Detector (SVD-2) used for these investigations consists of a precision silicon vertex detector, a drift tube tracker, a magnetic spectrometer with sixteen proportional chambers and a magnet, a Cherenkov counter and an electromagnetic calorimeter (EMCal). SVD-2 Collaboration (JINR, IHEP and SINP MSU) [వ] is aimed at the search for collective phenomena at high multiplicity area. It is known that mainly pions are produced at U-70 energies. Their mean energy decreases with multiplicity increase. In that system Bose-Einstein condensate (BEC) can be formed.

The theoretical and experimental studies of BEC are continuing from 70es. M. Gorenstein and V. Begun [B] have shown within the framework of an ideal pion gas model that sharp growth of the neutral pion number fluctuations will be a signal of BEC formation with the increase of total multiplicity (sum of the neutral and charged particle number). They proposed to measure a scaled variance, $\omega$, defined as the ratio of a variance of a neutral pion number distribution to their mean multiplicity $\omega=\left(<N_{0}^{2}>-<N_{0}>^{2}\right) /<N_{0}>$ at the fixed total multiplicity. In the thermodynamic limit [B] this quantity approaches to infinity. It reaches the finite value for the restricted system size formed in the collisions of two protons. Neutral pion number fluctuations were investigated in pp interactions at $50 \mathrm{GeV} / \mathrm{c}$ incident beam at U-70 versus total multiplicity, and the noticeable growth of $\omega$ with the total multiplicity increase have been revealed [2].

This study is carried out in two stages. At the first stage the topological cross sections are measured. At the second stage the neutral pion number distributions are restored. We could go down three orders on topological cross sections up to $\sim 10 \mathrm{nb}$ in the comparison with Mirabelle data [䧃]. Events with high charged multiplicity are extremely rare ones therefore we have designed a sophisticated trigger to suppress the recording of events with the multiplicity smaller than the given value, called a trigger level [[5].

The measured topological cross sections are corrected for trigger conditions, a detector acceptance, the efficiency of setup and the reconstruction algorithm. The measurements of charged multiplicity have been fulfilled with using of the silicon vertex detector. Comparison of the topological cross sections with models has shown that the negative binomial distribution (NBD) overestimates experimental data at high multiplicity region, $N_{c h}>20$, but describes well the region of a small multiplicity. Good agreement has been received using the gluon dominance model (GDM) with including the gluon source fission [可]. The neutral pion and photons multiplicities are determined by means of the electromagnetic calorimeter (EMCal).

First indications of the growth of the neutral pion number fluctuations at high total multiplicity area were obtained and published one year ago $[\square]$. Thereafter the number of analyzable events was increased two times. New more detail Monte Carlo simulation of apparatus performance has been carried out and the improvement of the photon reconstruction algorithm has been reached. Our preliminary conclusion remains in force with the more precise: the growth of a scaled variance is beginning from total multiplicity $N_{t o t}=18$ and gets 7 standard deviations at total multiplicity about 30 pions as opposed to the tendency for the simulated events. 

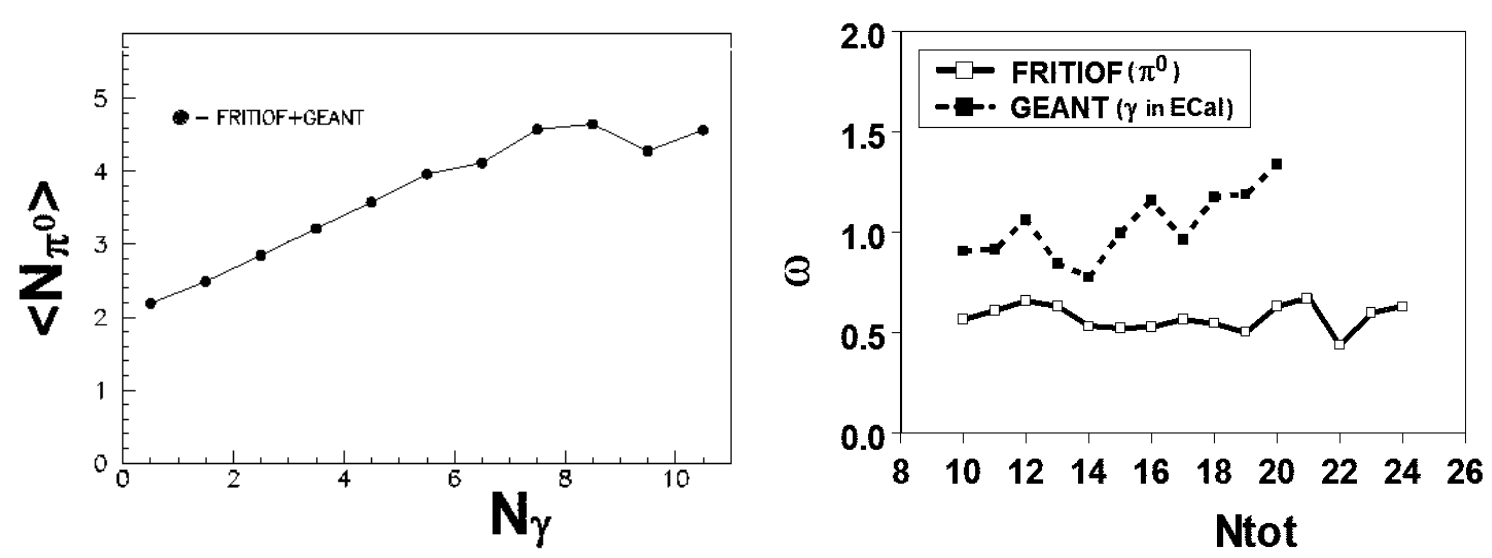

Figure 1: Monte Carlo code simulation. [Left] The mean multiplicity of neutral pions versus the photon number detected in EMCal. [Right] The scaled variance, $\omega$, versus $N_{\text {tot }}$ for photons $(\boldsymbol{\square})$ and $\pi^{0}$-mesons $(\square)$.

\section{Neutral pion restoration}

Owing to restricted aperture of EMCal and a threshold energy of a gamma-quantum registration, the restoration of a $\pi^{0}$ - mesons in every event is impossible. Their multiplicity is restored by means of Monte Carlo simulation. Now for this purpose we used Monte Carlo event generator FRITIOF7.02. For 10 mil simulated inelastic events the linear dependence between the mean multiplicity of produced $\pi^{0}$ - mesons and the number of the registered from their decays photons is corroborated (the left panel of Figure 1) again. Such dependence allows to determine a scaled variance of neutral pions indirectly by means of a measured scaled variance of $\gamma$ - quantum straight way. The scaled variance for Monte Carlo events is shown in the right panel of Figure 2. The small increasing of $\omega$ versus total multiplicity is observed for photons $\left(N_{t o t}=N_{c h}+N_{\gamma}\right)$ and at the same time its value are stayed the constant for neutral pions $\left(N_{t o t}=N_{c h}+N_{0}\right)$ in the investigated area.

To restore a neutral pion multiplicity Monte Carlo simulation (PYTHIA5.6, $10^{7}$ events) is used. These events we divide on the groups of events on the charged multiplicity, $N_{c h}$. Further we analyze of an every group. These groups consist of subgroups with the defined number of registered photons (observed in EMCal), $N_{\gamma}=\mathrm{i}$. It is obvious that almost of all these photons appear after decays of neutral pions (Monte Carlo simulation supports this conclusion).

Let $N_{e v}(i)$ is the number of all events with i registered photons and $N_{e v}(i, j)$ - the number of events found in the same subgroup and appeared after decay of $N_{0}=\mathrm{j}$ neutral pions. We define the matrix coefficients $c_{i j}=N_{e v}(i, j) / N_{e v}(i)$. These coefficients determine the share of events with $\mathrm{j}$ neutral pions among events registered as events with i photons. The simulation allows obtain $c_{i j}$ for $N_{\gamma} \leq 10$ and $N_{c h} \leq 14$ only. This is stipulated by this what the high multiplicity events are rare including Monte Carlo simulation even. Regularities of probabilities of $c_{i j}$ are used to extrapolate them to $N_{\gamma}>10$ and $N_{c h}>14$ region and the full sample of $N_{e v}\left(N_{t o t}, N_{c h}, N_{0}\right)$ is obtained.

Using these probabilities we can redistribute the number of experimental events with observing photon multiplicity into samples on the number of events with different multiplicity of $\pi^{0}$ - mesons. This is the inverse task. After that the neutral pion number distributions at the total multiplicity can be define. The average number of neutral pions $\left\langle N_{0}\right\rangle$ after their restoration (Fig. 2) is in 


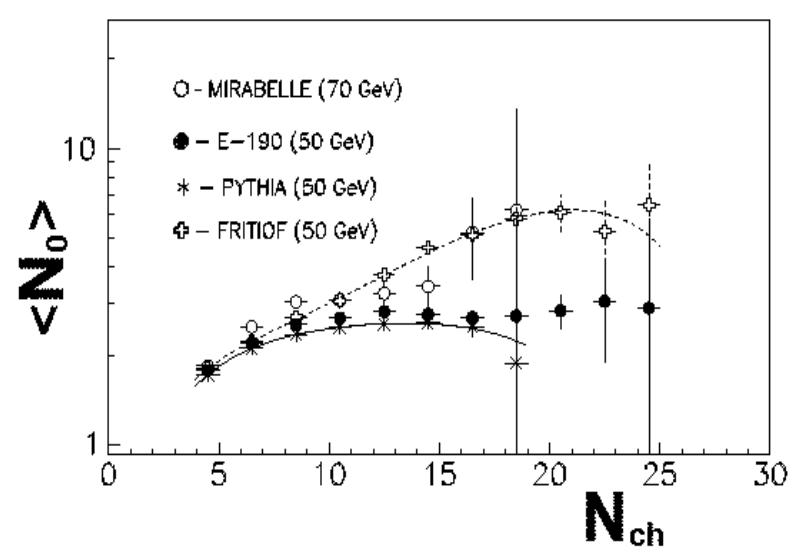

Figure 2: The dependence of average number of neutral pions $\left\langle N_{0}\right\rangle$ on charge multiplicity for Monte Carlo (PYTHIA, FRITIOF) events, Mirabelle and SVD-2 data.

the agreement with Mirabelle data at $70 \mathrm{GeV}$ [[]] (a small multiplicity region). This agreement testifies to the procedure of this restoration is carried out correctly. At high charged multiplicity the discrepancy between two Monte Carlo codes, PYTHIA6.2 and FRITIOF7.02 predictions are stipulated by the designed in these codes the different particle production mechanisms.

In present analysis about one million events of pp-interactions have been selected, two times more then at the previous one [प]. Photon reconstruction has been improved. It consisted of the searching for the cell consisted of $5 \times 5$ (in the previous case the $3 \times 3$ cell was chose) signal clusters in EMCal with analyzing of them with photon criteria. The transverse size of the glass is equal to the Molière radius. Besides, the next procedure is carried out. The experimental events were divided into groups according to charged particle number, $N_{c h}$. Every group is divided into subgroups of events on EMCal registered photon number, $N_{\gamma}$. In each such subgroup ( $N_{\gamma}$ is fixed) the number of events with certain number of $\pi^{0}$ - mesons is restored.

The transition from number of events with registered photon multiplicity to the number of events with restored neutral pion multiplicity is the inverse task. This task is decided with using of the $c_{i j}$ coefficients and the multiplicity distributions of neutral mesons, $N_{0}$ at different values of $N_{t o t}=N_{c h}+N_{0}$ are found. To analyze neutral pion number distributions at different values of the total multiplicity, $N_{t o t}$, the variable $n_{0}=N_{0} / N_{t o t}$, (a region of change [0,1]) is used. These distributions $r_{0}\left(n_{0}\right)$ are presented in the left panel of Figure 3 at $N_{t o t}=10,11, \ldots, 25+26+27$. The data for $N_{t o t}=25,26$, and 27 are combined due to small statistics.

\section{Neutral pion number fluctuations}

The distributions $r_{0}\left(n_{0}\right)$ in the left panel of Figure 3 are parameterized by Gauss function to find their mean value and standard deviation. After that the scaled variance is calculated. In the right panel of Figure 3 (top) the experimental, Monte Carlo simulation and model predictions of $\omega$ are presented. The experimental scaled variance is agreed well with the magnitude of $\omega$ defined on simulated events at $N_{t o t}<18$. At the same time we reveal the significant growth of its, reachable by more than 7 standard deviations at $N_{t o t} \simeq 30$ (the right panel of Figure 3, bottom) as opposed to the tendency for the simulated events. This growth has been observed both registered photons 

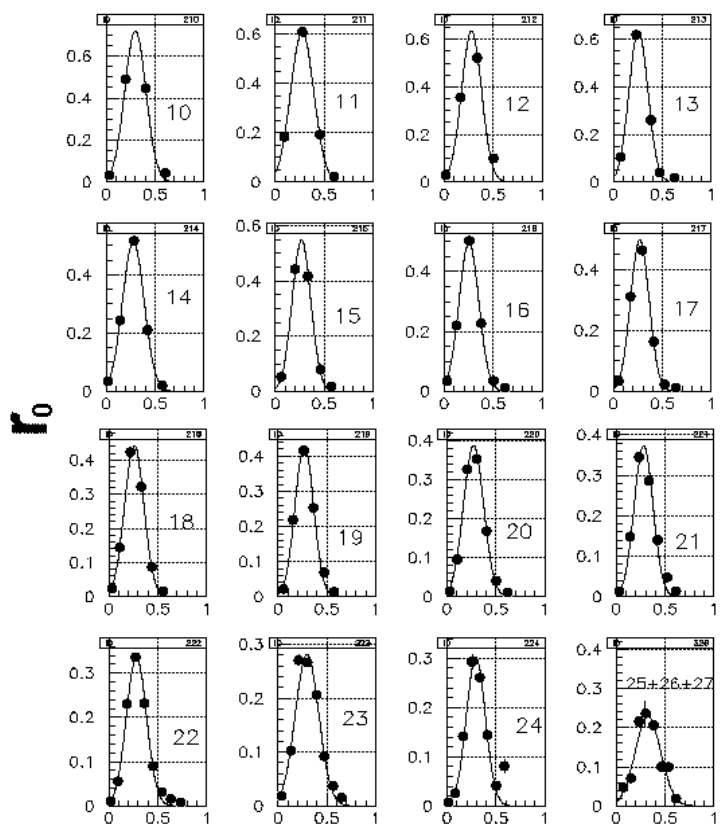

$\mathbf{n}_{0}$

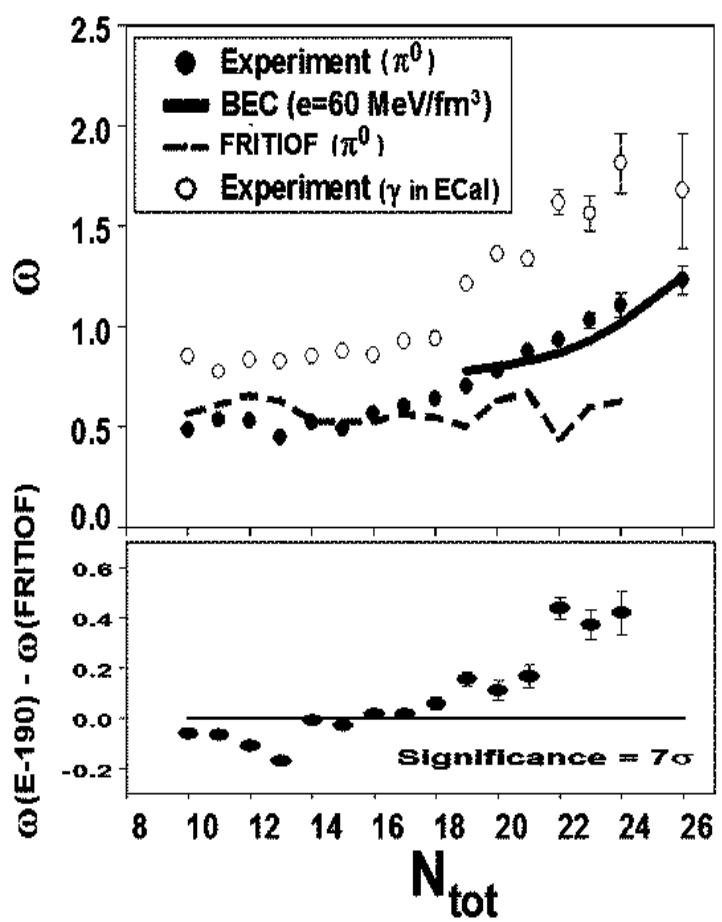

Figure 3: [Left] The neutral pion number distributions $r_{0}\left(n_{0}\right)$ versus scaled multiplicity, $n_{0}$ at difference values $N_{\text {tot }}(10,11, \ldots, 25+26+27)$. [Right] (Top) The measured scaled variance $\omega$ versus $N_{\text {tot }}$ for $\pi^{0}$-mesons $(\bullet)$, photons (०), MC code FRITIOF7.02 (the dashed curve) and theoretical prediction (solid curve) [B]] for energy density $\varepsilon=60 \mathrm{MeV} / \mathrm{fm}^{3} . N_{\text {tot }}=N_{c h}+N_{0}$ for $\pi^{0}$-mesons and $N_{t o t}=N_{c h}+N_{\gamma}$ for photons. (Bottom) The difference of experimental and simulated $\omega$ 's for $\pi^{0}$-mesons.

$\left(N_{t o t}=N_{c h}+N_{\gamma}\right)$, and restored neutral pions $\left(N_{t o t}=N_{c h}+N_{c h}\right)$. The theoretical predictions done by V. Begun and M. Gorenstein [B] at different conditions (the size of system, the energy density, the pion number density and others) at the high total pion multiplicity area testify to the Bose-Einstein condensate formation since we observe the growth of the scaled variance.

Critical point of pion condensation is determined in statistical physics: $E_{\text {crit }}=3.3\left(h^{2} / m_{\pi}\right) \rho^{2 / 3}$ [Q]. The density $\rho$ is equal to $0.2 \mathrm{fm}^{-3}$ if the interaction region size of two protons $\sim 3 \mathrm{fm}$ and $N_{t o t}=36$ (a maximal observable number of pions). In this case the critical energy is equal to $E_{c r i t}$ $\simeq 100 \mathrm{MeV}$. At $50-\mathrm{GeV}$ proton beam and $N_{\text {tot }}=30$ the mean energy of pion,

$$
E_{\pi}=\left(E_{c m s}-2 m_{n}-N_{t o t} m_{\pi}\right) / N_{\pi}
$$

is equal to $120 \mathrm{MeV}\left(m_{n}-\right.$ a nucleon mass, $N_{\pi}$ - a number of pions and $m_{\pi}-$ their mass). This value is compatible with $E_{\text {crit }}$. Thus the experimental observable growth of scaled variance at U-70 for registered $\gamma$-quanta and restored neutral pion multiplicity can testify to BEC formation in the pion system at high multiplicity events.

At present we are planning to study a soft photon $\left(E_{\gamma}<100 \mathrm{MeV}\right)$ yield versus neutral, charged and total multiplicities. The carried out in last tenths experiments point to the excess its yield in the comparison with the theoretical estimations. The exhaustive explanation of this collective phenomenon is absent. In the approach developed by S. Barshay [10] the excess of soft photon 
yield can be connected with BEC formation in the pion system. Also we will increase statistics to move forward to much higher multiplicity region.

I appreciate all participants of SVD-2 Collaboration for active and fruitful work. I also thank the ICHEPP 2012 Organizing Committee for exciting atmosphere and good conditions for the job and the communication during all stay time in Melbourne.

\section{References}

[1] CMS Collaboration, Observation of Long-Range Near-Side Angular Correlations in Proton-Proton Collisions at the LHC, JHEP 09 (2010) 091 [hep-ex/1009.4122].

[2] E. N. Ardashev et al. SVD-2 Collaboration. (in Russian) Proton interactions with high multiplicity. IHEP Preprint 2011-4 (2011) [hep-ex/1104.0101]. http://web.ihep.su/library/pubs/all-w.htm.; E. N. Ardashev et al. SVD-2 Collaboration. (in Russian) Neutral-Pion Fluctuations at High Multiplicity in pp Interactions at 50 GeV. IHEP Preprint 2011-5 (2011). [hep-ex/1104.3673] http://web.ihep.su/library/pubs/prep2011/11-5-w.htm.

[3] V. V. Begun and M. I. Gorenstein, Bose-Einstein condensation of pions in high multiplicity events, Phys. Lett. B 653 (2007) 190; V. V. Begun and M. I. Gorenstein, Bose-Einstein Condensation in the Relativistic Pion Gas: Thermodynamic Limit and Finite Size Effects. Phys. Rev. C77 (2008) 064903.

[4] V. V. Ammosov et al., Average charged particle multiplicity and topological cross-sections in 50-GeV/c and 69-GeV/c p p interactions. Phys. Lett. B 42 (1972) 519.

[5] A. Avdeichikov et al., A trigger of events with a high multiplicity of charged particles at the SVD-2 setup. Instrum. Exp. Tech. 2 (2011) 15.

[6] E. S. Kokoulina. Acta Phys. Polon. Description of pp interactions with very high multiplicity at 70-GeV/c. B 35 (2004) 295; Gluon dominance model. AIP Conf. Proc. 828 (2006) 81; E. A. Kuraev, S. Bakmaev, E. S. Kokoulina, Azimuthal correlation of gluon jets created in proton-antiproton annihilation. Nucl. Phys. B 851 (2011) 551.

[7] E. S. Kokoulina (On behalf of the SVD-2 Collaboration), Neutral Pion Fluctuations in pp Collisions at 50 GeV by SVD-2. Progr. Theor. Phys., 193 (2012) 306; V. N. Ryadovikov (On behalf of the SVD-2 Collaboration), Neutral-Pion Fluctuations at High Multiplicity in pp Interactions at $50 \mathrm{GeV}$. Phys. Atom. Nucl., 75 (2012) 989.

[8] M. Boratov et al. Gamma Production and Multiplicity Correlations Between Neutral and Charged Pions in p p Interactions at 69-GeV/c. Mirabelle Collaboration. Nucl. Phys. 111 (1976) 529.

[9] L. D. Landau and I. M. Lifshitz. Vol. 5. Statistical physics, part 1 (3ed., Pergamon, 1980).

[10] Saul Barshay, ANOMALOUS SOFT PHOTONS FROM A COHERENT HADRONIC PHASE IN HIGH-ENERGY COLLISIONS. Phys. Lett. B 227 (1989) 279. 